\title{
SOUVENIRS SUR QUELQUES ÉTAPES DU PARCOURS INTELLECTUEL D'ERNEST COUMET
}

\author{
Marc BARBUT
}

Triant récemment certaines de mes vieilles paperasses, je tombe sur deux feuillets manuscrits de notes que j'avais prises lors d'une séance de séminaire, rue Colbert. La suscription en est : «13.1.64. Coumez. Sur la notion de sort (la querelle des $-\mathrm{s}$ ). $16^{\mathrm{e}}-17^{\mathrm{e}} \mathrm{S}$. »

La faute d'orthographe sur le patronyme du conférencier montre que, selon toute vraisemblance, c'est ce lundi 13 janvier 1964 — et même vers 16 heures - qu'eut lieu ma première rencontre avec Ernest Coumet, pensionnaire à la fondation Thiers.

Un point de son exposé devait d'ailleurs faire peu après l'objet de sa première publication (il y en eut huit en tout) dans la revue Mathématiques et sciences humaines du Centre de mathématique sociale de l'École pratique des hautes études (EPHE) - VI ${ }^{\mathrm{e}}$ section : « Les jeux de hasard sont-ils une invention du diable? », Mathématiques et sciences humaines, 6, 1964.

Ainsi commença une longue amitié, et une collaboration constante de Coumet avec le Centre de mathématique sociale (CMS); collaboration qui s'inscrivait d'ailleurs avec bonheur dans le cadre plus général de celle qui existait déjà entre le CMS et le Centre Alexandre-Koyré (CAK), et dont la cohérence scientifique devait beaucoup à la communauté d'intérêts (et aussi de formation) d'hommes tels que Pierre Costabel, René Taton ou Georges-Th. Guilbaud.

En fait, Coumet avait déjà participé, quelques années plus tôt, à certaines activités du CMS ; mais sans le savoir. Je pense à cette année où, étudiant en philosophie à la faculté des lettres de l'université de Bordeaux, il suivait en compagnie de son ami Jean-Pierre Siméon le cours du professeur Roger Daval.

Ce dernier fréquentait assidûment à Paris (au 17 de la rue Richer) le $\mathrm{CMS}$, ses réunions et ses séminaires. Il y assistait, cette année-là, à des leçons données chaque semaine par Guilbaud autour du «problème des partis » de Blaise Pascal; il y prenait consciencieusement beaucoup de notes... dont bénéficiaient la semaine suivante les étudiants bordelais.

Revue de synthèse : $4^{\mathrm{e}}$ sér., $\mathrm{n}^{\text {os }}$ 2-3-4, avr.-déc. 2001, p. 287-290. 
Et c'est ainsi qu'un enseignement donné au CMS fut sans doute, pour partie tout au moins, à l'origine de l'attention qui devait se révéler si féconde par la suite, que Coumet a toujours portée à la «théorie du hasard », à sa naissance et à son histoire ultérieure. S'agit-il d'un hasard?

Commencée sous le signe de l'histoire du calcul des probabilités, cette collaboration se poursuivit et s'affirma en ce milieu des années 1960 sur un autre centre d'intérêt majeur de notre ami : l'histoire de la logique mathématique, et singulièrement de celle de son fondateur, George Boole.

Fin septembre 1965, le CMS organisa, à l'intention d'enseignants et de chercheurs en philosophie ou en psychologie principalement, un stage d'initiation aux Algèbres de Boole. Qui fut chargé du cours, qui eut lieu le lundi 27 septembre, sur l'œuvre de Boole lui-même? Ernest Coumet, bien sûr, qui publiera pendant l'année 1966 son grand texte «Logique, mathématique et langage dans l'œuvre de G. Boole », réparti sur trois livraisons de la revue Mathématiques et sciences humaines, ${ }^{\text {os }} 15,16$ et 17, 1966.

C'est également le moment de la parution, de concert avec Jean Gattégno, de sa traduction du livre de logique de Lewis Carroll.

L'année 1966 fut aussi celle du 250 anniversaire de la mort de Leibniz. À cette occasion, le Centre international de synthèse organisa du samedi 28 au lundi 30 mai (week-end de la Pentecôte! mais nous étions encore en un temps où les assemblées statutaires des directeurs d'études de l'EPHE se tenaient toujours un dimanche matin) trois «journées » consacrées à l'œuvre de Leibniz et à sa postérité.

Certains des participants à ce colloque trouvèrent que la place consacrée aux travaux mathématiques du grand Gottfried-Wilhelm y était insuffisante. Aussi le Centre Alexandre-Koyré (CAK), et le Centre de mathématique sociale organisèrent-ils en commun, sous la direction du R.P. Costabel, un complément à ces journées : la «Journée d'étude sur Leibniz et la mathématique », qui eut lieu le samedi 4 mars 1967 au 12 rue Colbert. C'est là qu'un exposé de Coumet révéla ce que fort peu de gens connaissaient alors : les travaux de Leibniz sur le «problème des partis ».

Parallèlement à ses recherches sur l'histoire du calcul des probabilités et sur celle de la logique mathématique, Coumet travailla beaucoup, dans ces mêmes années 1960, sur l'histoire de la combinatoire (et, corrélativement et accessoirement, celle de la cryptographie). C'est à cette époque qu'il prépara sa grande thèse : «Mersenne, Frenicle et l'élaboration de l'analyse combinatoire dans la première moitié du XVII ${ }^{\mathrm{e}}$ siècle ».

J'eus l'honneur, et le grand plaisir, d'être désigné, fin mars 1968, comme membre de son jury (selon la réglementation alors en vigueur, il y fallait une «potiche » pour représenter la faculté des lettres et sciences humaines de l'université de Paris). La soutenance était prévue pour le printemps 1968. Des événements, imprévus quant à eux, désorganisèrent les 
calendriers académiques, de sorte qu'elle n'eut lieu, très brillamment comme on peut s'en douter, que le 19 décembre 1968. Il était temps! Coumet était en effet au bout de sa période réglementaire de prise en charge par le CNRS et devait impérativement remplir les conditions nécessaires pour trouver un «point de chute» universitaire : celui-ci fut effectivement trouvé à la faculté des lettres de Nanterre, puis dans les années 1970, à l'université Paris-I (UER de philosophie). L'aboutissement : l'élection en 1977 à une direction d'études de l'École des hautes études en sciences sociales (Centre Alexandre-Koyré).

Vinrent les années 1980. Le milieu de la recherche en histoire des sciences y connut, à Paris notamment, quelques turbulences de nature institutionnelle. Turbulences très mal «vécues » par Coumet; à tel point que le Centre de mathématique sociale, qui aurait souhaité l'accueillir, lui avait déjà réservé une place (un éventuel refuge), pour le cas où les choses auraient trop mal tourné. Finalement, il n'en fut rien, fort heureusement.

Mais l'événement important de ces années fut l'initiative que prit notre ami de créer un séminaire consacré à l'histoire des «théories du hasard » et de leurs applications dans les sciences humaines. Patronnées conjointement par le CAK et le CMS, ces rencontres bimensuelles débutèrent au cours de l'année universitaire 1982-1983, sous le nom de "Séminaire d'histoire du calcul des probabilités »; les mots « ... et de la statistique » furent ajoutés quelques années plus tard à son intitulé. En fait, il traitait plus généralement de l'histoire et de l'épistémologie des méthodes mathématiques ou quantitatives dans les sciences sociales.

Nous trouvons parmi les conférenciers de la première année, Pierre Costabel, Georges-Th. Guilbaud, Bernard-Pierre Lécuyer et Bernard Bru qui y tiendra tant de place par la suite.

En près de vingt ans maintenant d'existence du séminaire, Coumet n'y apparaît que deux fois en tant que conférencier: le 17 mars 1989, sur l'arithmétique morale de Buffon, et le 7 mai 1999, sur le calcul des probabilités vu par Auguste Comte. Une fois tous les dix ans, cela peut sembler peu.

Mais la réalité est tout autre : avant chacune des séances, Coumet travaille le sujet traité; il arrive avec tout un dossier, fait de notes abondantes et de la documentation adéquate; il intervient longuement au cours de la discussion, apportant précisions, compléments ou vues originales dont tous les participants tirent profit; et souvent, en premier lieu, l'auteur lui-même de la communication discutée. Avec beaucoup de tact, mais fermement, Coumet a ainsi maintes fois mis en évidence les lacunes de l'information de tel ou tel d'entre nous. C'est de l'érudition et de la science récoltées au cours de décennies de lectures et de réflexions qu'il nous fait bénéficier. Avec quelle générosité! Car la préparation de chacune de ces séances lui 
coûte sûrement beaucoup en temps, et en efforts; surtout dans les périodes où il a dû lutter contre diverses infortunes physiques.

Tout ce travail qu'il nous a ainsi offert, au fil des années, a porté ses fruits : Ernest Coumet restera, pour le séminaire, l'un des deux maîtres qui ont le plus ensemencé de savoirs et d'idées les cervelles des fidèles (et aussi celles de moins fidèles) de nos réunions.

Marc BARBUT,

Centre d'analyse et de mathématique sociale, École des hautes études en sciences sociales, 54, bd Raspail, 75006 Paris (avril 2001).

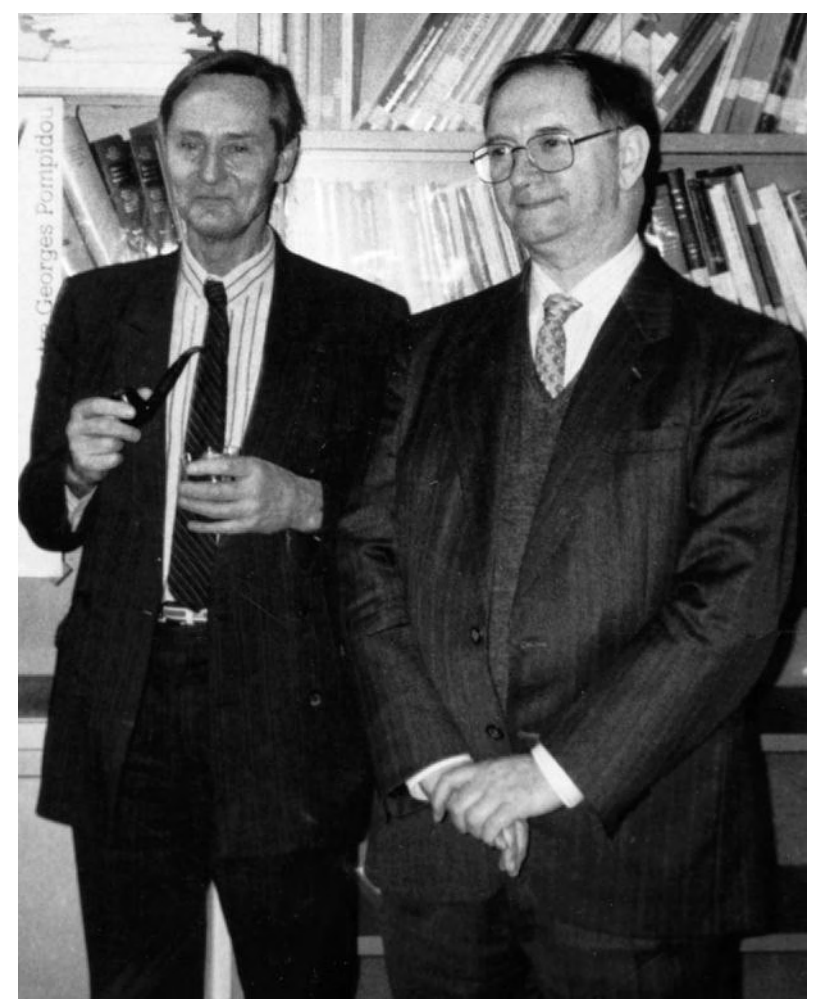

Marc Barbut et Ernest Coumet lors d'une remise de décoration à $M^{m e}$ M. Petruscewycz le lundi 7 janvier 1990

dans la bibliothèque du Centre de mathématique sociale à l'EHESS 